PROCEEDINGS OF THE

AMERICAN MATHEMATICAL SOCIETY

Volume 133, Number 7 , Pages 1993-2003

S 0002-9939(05)07854-8

Article electronically published on February 15, 2005

\title{
EXISTENCE AND MAPPING PROPERTIES OF THE WAVE OPERATOR FOR THE SCHRÖDINGER EQUATION WITH SINGULAR POTENTIAL
}

\author{
VLADIMIR GEORGIEV AND ANGEL IVANOV \\ (Communicated by Christopher D. Sogge)
}

\begin{abstract}
We consider the Schrödinger equation in three-dimensional space with small potential in the Lorentz space $L^{3 / 2, \infty}$ and we prove Strichartz-type estimates for the solution to this equation. Moreover, using Cook's method, we prove the existence of the wave operator. In the last section we prove the equivalence between the homogeneous Sobolev spaces $\dot{H}^{s}$ and $\dot{H}_{V}^{s}$ in the case $0 \leq s<\frac{3}{2}$.
\end{abstract}

\section{Definitions And Main RESults}

Consider the following Schrödinger equation with potential perturbation:

$$
\begin{gathered}
i \partial_{t} u-\Delta u+V u=0, \quad \Delta=\partial_{x_{1}}^{2}+\partial_{x_{2}}^{2}+\partial_{x_{3}}^{2}, \\
u(0, x)=u_{0}(x), \quad x \in \mathbb{R}^{3} .
\end{gathered}
$$

Here $V=V(x)$ is a real-valued potential that satisfies the assumption

$$
\|V\|_{L^{\left(\frac{3}{2}, \infty\right)}} \leq \delta_{0}
$$

where $L^{(p, q)}$ are standard Lorentz spaces and $L^{(p, \infty)}$ is the weak $L^{p}$ space (see [1] for details). For $\delta_{0}>0$ sufficiently small one can define the bilinear form

$$
Q(u, v)=(\nabla u, \nabla v)_{L^{2}\left(\mathbb{R}^{3}\right)}+\int_{\mathbb{R}^{3}} V(x) u(x) \overline{v(x)} \mathrm{d} x
$$

on $\dot{H}^{1}\left(\mathbb{R}^{3}\right)$ (see section 3). The Friedrichs extension of the quadratic form (see [15], [1] and [3]), implies that $-\triangle+V$ has dense domain $D=H^{2}\left(\mathbb{R}^{3}\right)$ such that, if $f \in D$, then $(-\triangle+V) f \in L^{2}$ and $V f \in L^{2}$. Moreover, $-\triangle+V$ with dense domain $D$ is a self-adjoint operator. The existence and mapping properties of the wave operators

$$
\Omega_{ \pm}=s^{-} \lim _{t \rightarrow \pm \infty} e^{i t H} e^{-i t H_{0}}
$$

Received by the editors February 16, 2004.

2000 Mathematics Subject Classification. Primary 35J10, 35P25, 35B45.

Key words and phrases. Schrödinger equation, Lorentz spaces, wave operators.

The authors were partially supported by the Research Training Network (RTN) HYKE, financed by the European Union, contract number: HPRN-CT-2002-00282. 
where $H_{0}=-\triangle, H=-\triangle+V$, are studied in [7]. The crucial assumption in [7], 8], [14 is that $V$ belongs to the Kato class (see for details (A.2) in [14] and the introduction in [7]). It is easy to see that a potential of type

$$
V(x)=\frac{W\left(\frac{x}{|x|}\right)}{|x|^{2}},
$$

where $W \in L^{\infty}\left(S^{2}\right)$, does not belong to Kato's class (for example, we can take $\left.W\left(\frac{x}{|x|}\right)=1\right)$.

Our main goal will be to prove the existence and mapping properties of the wave operator $\Omega_{ \pm}$for the larger class of potentials $V$, satisfying only (1.3). This problem is closely connected with another classical problem, namely the Strichartz-type estimates for the corresponding inhomogeneous Cauchy problem

$$
i \partial_{t} u-\Delta u=F, \quad u(0)=f .
$$

We shall call the pair $\left(\frac{1}{p}, \frac{1}{q}\right)$ sharp admissible (see 9 for this notion and the properties of sharp admissible pairs) if it satisfies the condition:

$$
\frac{3}{4}=\frac{1}{p}+\frac{3}{2 q}, \quad 2 \leq p \leq \infty .
$$

Our main result is given in the next theorem.

Theorem 1.1. If $(p, q)$ and $(\tilde{p}, \tilde{q})$ satisfy (1.8), then the solution to the Cauchy problem

$$
i \partial_{t} u-\triangle u+V u=F,(t, x) \in \mathbb{R}_{t} \times \mathbb{R}_{x}^{3}, \quad u(0, x)=f(x),
$$

satisfies the estimate:

$$
\|u\|_{L^{p}\left(\mathbb{R}_{t} ; L_{x}^{(q, 2)}\right)}+\|u\|_{C\left(\mathbb{R}_{t} ; L^{2}\right)} \leq C\left(\|F\|_{L^{\tilde{p}^{\prime}}\left(\mathbb{R}_{t} ; L_{x}^{\left(\tilde{q}^{\prime}, 2\right)}\right)}+\|f\|_{L^{2}}\right) .
$$

Similar Strichartz type estimates have been discussed in 6 for the case of a potential decaying rapidly at infinity. In [12] the case of potentials in the Kato class is discussed. Since the Kato class is smaller than the Lorentz space $L^{3 / 2, \infty}$, it is clear that the above result enables one to treat all small $L^{3 / 2, \infty}$ potentials outside the Kato class, in particular potentials of type (1.6). The case of the inverse square potential is considered in [2], where similar Strichartz type estimates are established under natural assumptions on the negative part of the potential.

The main idea is to check (1.10) for the endpoints $(p, q)=(2,6)$ and $(p, q)=$ $(2, \infty)$ of the interval $A E$, where $E=\left(\frac{1}{2}, \frac{1}{6}\right), A=\left(0, \frac{1}{2}\right)$. Let us set $L_{t}^{p} L_{x}^{(q, r)}=$ $L^{p}\left(\mathbb{R}_{t} ; L_{x}^{(q, r)}\right)$. Then we have to verify the inequality

$$
\|u\|_{L_{t}^{2} L_{x}^{6}} \leq C\|F\|_{L_{t}^{2} L_{x}^{\frac{6}{5}}}
$$

where $u$ is a solution of the Schrödinger equation with zero initial data. A stronger version of (1.11), namely

$$
\|u\|_{L^{2} L^{(6,2)}} \leq C\|F\|_{L^{2} L^{\left(\frac{6}{5}, 2\right)}}
$$

is valid for the case $V=0$ (see [9]). We can obtain the same estimate using the Hölder inequality in Lorentz spaces (see Lemma 4.2 in [13]) and the assumption (1.3). 
Our first goal as an application of the previous result is to obtain an existence of the weak limit in $L^{2}$ :

$$
\Omega_{ \pm}=w-\lim _{t \rightarrow \pm \infty} e^{i t H} e^{-i t H_{0}} .
$$

Theorem 1.2. If the potential $V \in L^{\left(\frac{3}{2}, \infty\right)}$ satisfies the assumption (1.3) with $\delta_{0}>0$ sufficiently small, then for every $f \in L^{2}$ there exists the weak limit

$$
\lim _{t \rightarrow \pm \infty} U(t) U_{0}(-t) f
$$

in $L^{2}$ and the limit is unique.

The following a priori estimate,

$$
\int_{0}^{T}\left\langle f, U(\tau) V U_{0}(-\tau) g\right\rangle_{L^{2}} \mathrm{~d} \tau \leq C\|f\|_{L^{2}}\|g\|_{L^{2}},
$$

is the main step in the proof of Theorem 1.2 Our proof of (1.15) is based on the application of the Strichartz estimate of Theorem 1.1. The existence of the strong limit

$$
\Omega_{ \pm}=s-\lim _{t \rightarrow \pm \infty} e^{i t H} e^{-i t H_{0}}
$$

is obtained under a stronger assumption on $V$, namely

$$
\left\||x|^{a} V\right\|_{L^{(q, \infty)}}<\infty, \quad \frac{1}{q}=\frac{2-a}{3},
$$

where $\frac{1}{2}<a<2$. It is obvious that if the potential $V$ has the form (1.6), then (1.17) is satisfied.

Theorem 1.3. If the assumptions (1.3) (1.17) are satisfied, then for every $f \in L^{2}$ there exists the strong limit

$$
\lim _{t \rightarrow \pm \infty} U(t) U_{0}(-t) f
$$

The argument of the proof of this result is similar to the proof of Theorem 1.2 Finally, we are looking for a maximal interval $s \in\left[0, s_{0}\right)$ such that

$$
\Omega_{ \pm}: H^{s} \rightarrow H^{s} .
$$

The key point is to verify the equivalence $\dot{H}_{V}^{s}=\dot{H}^{s}$ for $s \in\left[0, s_{0}\right)$, where $\dot{H}_{V}^{s}$ is the closure of $D\left((-\Delta+V)^{\frac{s}{2}}\right)$ with respect to the norm $\left\|(-\Delta+V)^{\frac{s}{2}} f\right\|_{L^{2}}$. From Theorem 1.3 it follows that $\Omega_{ \pm}: L^{2} \rightarrow L^{2}$. If we prove that $\dot{H}_{V}^{s}=\dot{H}^{s}$, then immediately we may deduce (1.19). We have the following.

Theorem 1.4. If $V$ satisfies (1.3) and $\delta_{0}$ is small enough, then $\dot{H}_{V}^{s}=\dot{H}^{s}$ for $0 \leq s<\frac{3}{2}$.

\section{FRIEDRICHS EXTENSION OF $\triangle_{V}=\triangle-V$}

Let us consider the quadratic form

$$
B(f, f)=(\nabla f, \nabla f)_{L^{2}\left(\mathbb{R}^{3}\right)}+\int_{\mathbb{R}^{3}} V(x)|f(x)|^{2} \mathrm{~d} x .
$$

In this case we can use the KLMN theorem (see theorem 10.17 in [11] vol.2). Due to this theorem it is sufficient to verify the estimate

$$
\int_{\mathbb{R}^{3}} V(x)|f(x)|^{2} \mathrm{~d} x \leq a \int_{\mathbb{R}^{3}}|\nabla f(x)|^{2} \mathrm{~d} x+b\|f\|_{L^{2}\left(\mathbb{R}^{3}\right)}^{2}
$$


with $a<1$ and $f \in H^{1}$. Indeed, the assumption (1.3) and the Hölder inequality in Lorentz spaces (see Theorem 3.4 in [10]) imply that

$$
\|\sqrt{|V|} f\|_{L^{2}} \leq C\|\sqrt{|V|}\|_{L^{(3, \infty)}}\|f\|_{L^{(6,2)}} \leq C \delta_{0}\|f\|_{L^{(6,2)}} .
$$

Using the Sobolev embedding (see [1]) $\dot{H}^{1}\left(\mathbb{R}^{3}\right) \subset L^{(6,2)}\left(\mathbb{R}^{3}\right)$, we get $\|f\|_{L^{(6,2)}} \leq$ $C_{1}\|f\|_{\dot{H}^{1}}$, so

$$
\int V(x)|f(x)|^{2} \mathrm{~d} x=\|\sqrt{|V|} f\|_{L^{2}}^{2} \leq C^{2} \delta_{0}^{2} C_{1}^{2}\|\nabla f(x)\|_{L^{2}}^{2} .
$$

If $\delta_{0}$ is such that $2 \delta_{0} C_{1}<1$, i.e. $\delta_{0}<1 /\left(C C_{1}\right)=1 /\left(2 C_{1}\right)$, where $C=2$ is the constant from the Hölder inequality (see [10, Theorem 3.4) and $C_{1}$ is the constant from the Sobolev embedding, then we can conclude due to the KLMN theorem that there exists a self-adjoint operator $-\triangle+V$, such that

$$
((-\triangle+V) f, f)_{L^{2}}=\|\nabla f(x)\|_{L^{2}}^{2}+\int V(x)|f(x)|^{2} \mathrm{~d} x .
$$

(Note that we can take $b=0$ in $(2.2)$ and $((-\Delta+V) f, f) \geq 0$ for sufficiently small $\left.\delta_{0}.\right)$

\section{Strichartz ESTIMATE FOR The SChrÖDINGER EQUATION}

Using the Friedrichs extension of $\triangle_{V}=\Delta-V$ we can represent the solution to (1.1) as

$$
u(t)=U(t) u_{0}, \quad U(t)=e^{-i t \triangle_{V}} .
$$

Since $\triangle_{V}$ is self-adjoint, $U(t)$ is a unitary group in $L^{2}$ and we have the classical conservation of the charge,

$$
\left\|U(t) u_{0}\right\|_{L^{2}}=\left\|u_{0}\right\|_{L^{2}} .
$$

Our next step is to establish the endpoint estimate for (1.9); namely, we shall show that

$$
\|u\|_{L_{t}^{2} L_{x}^{6}} \leq C\|F\|_{L_{t}^{2} L_{x}^{\frac{6}{5}}} \cdot
$$

Indeed, we can rewrite the equation in (1.9) as follows:

$$
\begin{gathered}
i \partial_{t} u-\triangle u=F_{1}, \\
u(0, x)=0,
\end{gathered}
$$

where $F_{1}=F-V u$. Applying the Strichartz estimate for (3.4) and (3.5) we have that the inequality

$$
\|u\|_{L_{t}^{2} L_{x}^{(6,2)}} \leq C_{2}\left\|F_{1}\right\|_{L_{t}^{2} L_{x}^{\left(\frac{6}{5}, 2\right)}}
$$

is satisfied (see [9]). Since

$$
\left\|F_{1}\right\|_{L_{t}^{2} L_{x}^{(6,2)}} \leq\|F\|_{L_{t}^{2} L_{x}^{\left(\frac{6}{5}, 2\right)}}+\|V u\|_{L_{t}^{2} L_{x}^{\left(\frac{6}{5}, 2\right)}},
$$

we are in a position to apply the Hölder estimate:

$$
\|V f\|_{L^{\left(\frac{6}{5}, 2\right)}} \leq 2\|V\|_{L^{\left(\frac{3}{2}, \infty\right)}}\|u\|_{L^{(6,2)}} \leq 2 \delta_{0}\|u\|_{L^{(6,2)}} .
$$


If $\delta_{0}$ is such that $2 \delta_{0} C_{2}<1\left(C_{2}\right.$ is the constant in (3.6) $)$, then from (3.6), (3.7) and (3.8) we see that

$$
\|u\|_{L_{t}^{2} L_{x}^{6}} \leq \frac{C_{2}}{1-2 \delta_{0} C_{2}}\|F\|_{L_{t}^{2} L_{x}^{\frac{6}{5}}} .
$$

Using the theorem of Calderón (see for instance Lemma 2.5 in [10]),

$$
\|u\|_{L^{(p, d)}} \leq\left(\frac{d_{1}}{p}\right)^{\frac{1}{d_{1}}-\frac{1}{d}}\|u\|_{L^{\left(p, d_{1}\right)}}
$$

for $d>d_{1}, \quad 1<p<\infty$, we get

$$
\|u\|_{L_{t}^{2} L_{x}^{6}}=\|u\|_{L_{t}^{2} L_{x}^{(6,6)}} \leq\left(\frac{1}{3}\right)^{\frac{1}{3}}\|u\|_{L_{t}^{2} L_{x}^{(6,2)}}
$$

and

$$
\|F\|_{L_{t}^{2} L_{x}^{\frac{6}{5}}}=\|F\|_{L_{t}^{2} L_{x}^{\left(\frac{6}{5}, \frac{6}{5}\right)}} \geq\|F\|_{L_{t}^{2} L_{x}^{\left(\frac{6}{5}, 2\right)}}
$$

so we arrive at

$$
\|u\|_{L_{t}^{2} L_{x}^{6}} \leq C_{2}\left(\frac{1}{3}\right)^{\frac{1}{3}}\|F\|_{L_{t}^{2} L_{x}^{\frac{6}{5}}}
$$

In order to obtain the estimate

$$
\|u\|_{L_{t}^{2} L_{x}^{6}} \leq C\|F\|_{L_{t}^{1} L_{x}^{2}}
$$

we will decompose the function $u$ into two parts, i.e. $u=u_{1}+u_{2}$, where the functions $u_{1}$ and $u_{2}$ are solutions to the following equations:

$$
\begin{gathered}
i \partial_{t} u_{1}-\Delta u_{1}=F, \\
u_{1}(0)=0 ;
\end{gathered}
$$

and respectively

$$
\begin{gathered}
i \partial u_{2}-\Delta u_{2}=-V u, \\
u_{2}(0)=0 .
\end{gathered}
$$

Using the estimates $\left\|u_{1}\right\|_{L_{t}^{2} L_{x}^{6,2}} \leq C\|F\|_{L_{t}^{1} L_{x}^{2}}$ and $\left\|u_{2}\right\|_{L_{t}^{2} L_{x}^{6,2}} \leq C\|V u\|_{L_{t}^{2} L_{x}^{\frac{6}{5}, 2}} \leq$ $C \delta_{0}\|u\|_{L_{t}^{2} L_{x}^{6,2}}$, we may conclude that the following estimate for $u$ is true:

$$
\|u\|_{L_{t}^{2} L_{x}^{6,2}} \leq C\left(\|F\|_{L_{t}^{1} L_{x}^{2}}+\delta_{0}\|u\|_{L_{t}^{2} L_{x}^{6,2}}\right) .
$$

Hence, for sufficiently small $\delta_{0}$, we have $\|u\|_{L_{t}^{2} L_{x}^{6,2}} \leq C\|F\|_{L_{t}^{1} L_{x}^{2}}$. The obvious estimate $\|u\|_{L_{t}^{2} L_{x}^{6}} \leq C\|u\|_{L_{t}^{2} L_{x}^{6,2}}$ gives the desired result.

Further, we have the following energy estimate for (1.9):

$$
\|u\|_{L_{t}^{\infty} L_{x}^{2}} \leq C\|F\|_{L_{t}^{1} L_{x}^{2}} .
$$

Turning to the estimate

$$
\|u\|_{L_{t}^{\infty} L_{x}^{2}} \leq C\|F\|_{L_{t}^{2} L_{x}^{\frac{6}{5}}}
$$

we put $V u$ on the right-hand side of the equation and use the free Strichartz estimate $\|u\|_{L^{\infty} L^{2}} \lesssim\|F-V u\|_{L^{2} L^{\frac{6}{5}}} \lesssim\|F\|_{L^{2} L^{\frac{6}{5}}}+\|V u\|_{L^{2} L^{\frac{6}{5}}} \lesssim\|F\|_{L^{2} L^{\frac{6}{5}}}+\|u\|_{L^{2} L^{6}}$. Now 
from the inequality (3.13) we obtain (3.21). Interpolating between the estimate (3.21) and the estimate (3.20), we get

$$
\|u\|_{L_{t}^{\infty} L_{x}^{2}} \leq C\|F\|_{L_{t}^{\tilde{p}^{\prime}} L_{x}^{\tilde{q}^{\prime}}}
$$

for any admissible pair $(\tilde{p}, \tilde{q})$. On the other hand, interpolation between the estimates (3.13) and (3.14) gives us

$$
\|u\|_{L_{t}^{2} L_{x}^{6}} \leq C\|F\|_{L_{t}^{\tilde{p}^{\prime}} L_{x}^{\tilde{q}^{\prime}}}
$$

Thus we can conclude that

$$
\|u\|_{L_{t}^{p} L_{x}^{q}} \leq C\|F\|_{L_{t}^{\tilde{p}^{\prime}} L_{x}^{\tilde{q}^{\prime}}}
$$

Using the $T T^{*}$ argument due to [4, we get also the estimate

$$
\|u\|_{L_{t}^{p} L_{x}^{q}} \leq C\left\|u_{0}\right\|_{L^{2}}
$$

for the solution of (1.9). Thus Theorem 1.1 is proved.

Corollary 3.1. Under the assumptions of Theorem 1.1 we have

$$
\begin{aligned}
& \|u\|_{L^{p}\left([0, T] ; L_{x}^{(q, 2)}\right)}+\|u\|_{C\left([0, T] ; L^{2}\right)} \\
\leq & C\|F\|_{L^{\tilde{p}^{\prime}}\left([0, T] ; L_{x}^{\left(\tilde{q}^{\prime}, 2\right)}\right)}+C\|f\|_{L^{2}} .
\end{aligned}
$$

\section{Existence of the WaVe operator}

Let $U(t)=e^{-i t \Delta_{V}}$ and $U_{0}(t)=e^{-i t \triangle}$ be the perturbed and free propagators respectively.

Proof of Theorem 1.2. The proof is based on Cook's method (see [11], vol.3), i.e. we shall use the relation

$$
U(t) U_{0}(-t)-U(s) U_{0}(-s)=i \int_{s}^{t} U(\tau) V U_{0}(-\tau) \mathrm{d} \tau .
$$

To show the existence of the limit (1.14), it is sufficient to show that for any couple $f, g \in L^{2}$ we have the estimate

$$
\int_{0}^{T}\left\langle f, U(\tau) V U_{0}(-\tau) g\right\rangle_{L^{2}} \mathrm{~d} \tau \leq C\|f\|_{L^{2}}\|g\|_{L^{2}}
$$

with constant $C$ independent of $T, f, g$. Indeed, the property (4.2) implies that for every sequence $t_{n} \uparrow \infty$ the elements $\psi_{n}=U\left(t_{n}\right) U_{0}\left(-t_{n}\right) f \in L^{2}$ have a weak limit $\psi_{*} \in L^{2}$. The inequality (4.2) enables us to show the existence of the weak limit (4.1). Indeed, for some other sequence $t_{n}^{\prime} \uparrow \infty$ we have $\psi_{n}^{\prime}=U\left(t_{n}^{\prime}\right) U_{0}\left(-t_{n}^{\prime}\right) f \in L^{2}$ and $\psi_{n}^{\prime}$ has a weak limit $\psi_{* *} \in L^{2}$. Then $\left(\psi_{*}, \psi_{n}\right) \rightarrow\left\|\psi_{*}\right\|^{2}$ and $\left(\psi_{*}, \psi_{n}^{\prime}\right) \rightarrow$ $\left(\psi_{*}, \psi_{* *}\right)$. On the other hand, (4.2), (4.1) show that $\left(\psi_{*}, \psi_{n}\right)-\left(\psi_{*}, \psi_{n}^{\prime}\right)$ is small as $n \rightarrow \infty$, so

$$
\left\|\psi_{*}\right\|^{2}=\left(\psi_{*}, \psi_{* *}\right)_{L^{2}}
$$

and in a similar way we get

$$
\left\|\psi_{* *}\right\|^{2}=\left(\psi_{*}, \psi_{* *}\right)_{L^{2}} .
$$


Combining (4.3) and (4.4), one obtains $\psi_{*}=\psi_{* *}$, and it remains to show (4.2). To verify (4.2), we rewrite the integral on the left-hand side of (4.2) as

$$
\begin{aligned}
& \int_{0}^{T}\left|\left\langle\sqrt{|V|} U(-\tau) f, \operatorname{sign}(V) \sqrt{|V|} U_{0}(-\tau) g\right\rangle_{L^{2}}\right| \mathrm{d} \tau \\
& \leq \int_{0}^{T}\|\sqrt{|V|} U(-\tau) f\|_{L^{2}}\left\|\sqrt{|V|} U_{0}(-\tau) g\right\|_{L^{2}} \mathrm{~d} \tau
\end{aligned}
$$

so the Cauchy inequality in $\tau$ implies that we have to show

$$
\begin{gathered}
\int_{0}^{T}\|\sqrt{|V|} U(-\tau) f\|_{L^{2}}^{2} \mathrm{~d} \tau \leq C\|f\|_{L^{2}}^{2}, \\
\int_{0}^{T}\left\|\sqrt{|V|} U_{0}(-\tau) g\right\|_{L^{2}}^{2} \mathrm{~d} \tau \leq C\|g\|_{L^{2}}^{2} .
\end{gathered}
$$

It is sufficient to show only one of them, for example the first one. To this end, we note that $\sqrt{|V|} \in L^{(3, \infty)}$, so applying the Hölder inequality for Lorentz spaces, we get

SO

$$
\|\sqrt{|V|} U(-\tau) f\|_{L^{2}} \leq C\|\sqrt{|V|}\|_{L^{(3, \infty)}}\|U(-\tau) f\|_{L^{(6,2)}}
$$

$$
\begin{gathered}
\int_{0}^{T}\|\sqrt{|V|} U(-\tau) f\|_{L^{2}}^{2} \mathrm{~d} \tau \\
\leq C \int_{0}^{T}\|U(-\tau) f\|_{L^{(6,2)}}^{2} \mathrm{~d} \tau=C\|U(-\tau) f\|_{L^{2}\left([0, T] ; L^{(6,2)}\right)}^{2} .
\end{gathered}
$$

Applying the Strichartz estimate (1.10), we get $\|U(-\tau) f\|_{L^{2}\left([0, T] ; L^{(6,2)}\right)}^{2} \leq\|f\|_{L^{2}}$. This proves (4.6) and completes the proof of the theorem.

Proof of Theorem 1.3. Since $U(t)$ and $U_{0}(t)$ are unitary operators in $L^{2}$, it is sufficient to show the existence of a strong limit in (1.18) for $f \in D$, where $D \subset L^{2}$ is a dense domain. For example, we could take

$$
D=\left\{f \in L^{2}: \operatorname{supp} f \subset\{|x| \leq R\}\right\} .
$$

We shall show in this case that there exists $C=C(R)$ so that

$$
\int_{1}^{T}\left\|U(\tau) V U_{0}(-\tau) f\right\|_{L^{2}} \mathrm{~d} \tau \leq C\|f\|_{L^{2}}
$$

It is clear that this estimate implies (1.18). Since $U(\tau)$ is a unitary operator in $L^{2}$, (4.11) follows from

$$
\int_{0}^{T}\left\|V U_{0}(-\tau) f\right\|_{L^{2}} \mathrm{~d} \tau \leq C\|f\|_{L^{2}} .
$$

To this end we apply the Hölder inequality, combined with (1.17) and get

$$
\left\|V U_{0}(-\tau) f\right\|_{L^{(2,2)}} \leq\left\||x|^{a} V\right\|_{L^{(q, \infty)}}\left\||x|^{-a} U_{0}(-\tau) f\right\|_{L^{(p, 2)}},
$$

where

$$
\frac{1}{q}=\frac{2-a}{3}, \frac{1}{p}=\frac{1}{2}-\frac{1}{q}=-\frac{1}{6}+\frac{a}{3} .
$$


For $a \in(0,2)$ it is clear that $-\frac{1}{6}+\frac{a}{3} \in(0,1)$ is equivalent to $a>\frac{1}{2}$. Now one can show that the limit in (4.1) is strong using the Kato - Jensen inequality (see [5])

$$
\left\||x|^{-2} U_{0}(\tau) f\right\|_{L^{2}} \leq \frac{C}{\tau^{2}}\left\||x|^{2} f\right\|_{L^{2}} .
$$

Interpolation between this inequality and the classical dispersive estimate for $U_{0}(\tau)$,

$$
\left\|U_{0}(\tau) f\right\|_{L^{\left(p_{0}, 2\right)}} \leq \frac{C}{\tau^{\frac{3}{2}-\frac{3}{p_{0}}}}\|f\|_{L^{\left(p_{0}^{\prime}, 2\right)}}, 2 \leq p_{0} \leq \infty
$$

gives us

$$
\begin{gathered}
\left\||x|^{-a} U_{0}(\tau) f\right\|_{L^{(p, 2)}} \leq \frac{C}{\tau^{a+\frac{3}{2}-\frac{3}{p}}}\left\||x|^{a} f\right\|_{L^{\left(p^{\prime}, 2\right)}}, \\
2 \leq p<\infty \quad, \quad 0 \leq a \leq 2 .
\end{gathered}
$$

Now applying (4.17), we get

$$
\int_{1}^{T}\left\||V| U_{0}(-\tau) f\right\|_{L^{2}} \mathrm{~d} \tau \leq \int_{1}^{t} \frac{1}{\tau^{a+\frac{3}{2}-\frac{3}{p}}}\left\||x|^{a} f\right\|_{L^{p^{\prime}}} \mathrm{d} \tau .
$$

Since suppf $\subset\{|x| \leq R\}$ and $p^{\prime}<2$, we get $\left\||x|^{a} f\right\|_{L^{p^{\prime}}} \leq C(R)\|f\|_{L^{2}}$ and we need only the condition $a+\frac{3}{2}-\frac{3}{p}>1$ for some $a, p$ satisfying (4.14). The last inequality is equivalent to $a+\frac{3}{2}-\frac{3}{p}-a>1 \Leftrightarrow 2>1$. Since this inequality is obvious, the condition is satisfied for $2>a>\frac{1}{2}$. Hence (4.18) shows that (4.12) is fulfilled. This completes the proof.

\section{Equivalence of $\dot{H}_{V}^{s}$ AND $\dot{H}^{s}$ FOR $n=3$}

To show that $\dot{H}_{V}^{s}=\dot{H}^{s}$ for $s<\frac{3}{2}$ we will prove the following.

Lemma 5.1. $\dot{H}_{V}^{1}=\dot{H}^{1}$.

Proof. It is sufficient to apply the argument of section 2.

Corollary 5.1. $(-\triangle+V)^{1 / 2}: \dot{H}^{1} \rightarrow L^{2}$ and $(-\triangle+V)^{s / 2}: \dot{H}^{s} \rightarrow L^{2}$ for $0 \leq s \leq$ 1.

Further we need the following.

Lemma 5.2. $\left\||V|^{s / 2} f\right\|_{L^{2}} \leq C\|f\|_{\dot{H}^{s}}$ for $0 \leq s<\frac{3}{2}$.

Proof. Applying the Hölder inequality for Lorentz spaces and using the fact that $\left\||V|^{s / 2}\right\|_{L^{\left(\frac{3}{s}, \infty\right)}} \leq C\|V\|_{L^{\left(\frac{3}{2}, \infty\right)}}^{s / 2} \leq C_{0}^{s / 2}$, we get

$$
\begin{gathered}
\left\||V|^{s / 2} f\right\|_{L^{2}} \leq C\left\||V|^{s / 2}\right\|_{L^{\left(\frac{3}{s}, \infty\right)}}\|f\|_{L^{(q, 2)}} \\
\frac{1}{2}=\frac{s}{3}+\frac{1}{q}, \quad q=6 \in(2, \infty)
\end{gathered}
$$

Now we can apply the Sobolev embedding (see [1]) $\dot{H}^{s} \subset L^{(q, 2)}$ for $\frac{1}{2}=\frac{s}{3}+\frac{1}{q}$, and we get $\left\||V|^{s / 2} f\right\|_{L^{2}} \leq C \delta_{0}^{s / 2} C_{1}\|f\|_{\dot{H}^{s}}$. 
Proof of Theorem 1.4. Take $1<s<\frac{3}{2}$. We shall use the identity

$$
\begin{gathered}
\left\|(-\Delta+V)^{s / 2} f\right\|_{L^{2}}^{2}=\left((-\triangle+V)^{s-1} f,(-\triangle+V) f\right)_{L^{2}} \\
=\left((-\triangle+V)^{s-1} f,(-\triangle) f\right)_{L^{2}}+\left((-\triangle+V)^{s-1} f, V f\right)_{L^{2}} \\
=\left((-\triangle)^{1-s / 2}(-\triangle+V)^{s-1} f,(-\triangle)^{s / 2} f\right)_{L^{2}} \\
+\left(|V|^{1-s / 2}(-\triangle+V)^{s-1} f, \operatorname{sgn} V|V|^{s / 2} f\right)_{L^{2}} .
\end{gathered}
$$

Let we set

$$
\begin{aligned}
& I_{1}=\left((-\triangle)^{1-s / 2}(-\triangle+V)^{s-1} f,(-\triangle)^{s / 2} f\right)_{L^{2}}, \\
& I_{2}=\left(|V|^{1-s / 2}(-\triangle+V)^{s-1} f, \operatorname{sgn} V|V|^{s / 2} f\right)_{L^{2}} .
\end{aligned}
$$

Now we can apply the second conclusion of Corollary 5.1 and, using the fact that $\frac{1}{2}<2-s<1$, we get $\left\|(-\triangle)^{(2-s) / 2} g\right\|_{L^{2}} \leq C\left\|(-\triangle+V)^{(2-s) / 2} g\right\|_{L^{2}}$. Now taking $g=(-\triangle+V)^{s-1} f$, we get

$$
\left\|(-\triangle)^{(2-s) / 2}(-\triangle+V)^{s-1} f\right\|_{L^{2}} \leq C\left\|(-\triangle+V)^{s / 2} f\right\|_{L^{2}} .
$$

Hence

$$
\left|I_{1}\right| \leq\|f\|_{\dot{H}_{V}^{s}}\|f\|_{\dot{H}^{s}} .
$$

Now we are ready to estimate the term $I_{2}$. We have

$$
\left|I_{2}\right| \leq\left\||V|^{(2-s) / 2}(-\triangle+V)^{s-1} f\right\|_{L^{2}}\left\|(V)^{s / 2} f\right\|_{L^{2}} .
$$

Since $2-s \in\left(0, \frac{3}{2}\right)$, we can apply Lemma 5.2 and get

$$
\left\||V|^{(2-s) / 2}(-\triangle+V)^{s-1} f\right\|_{L^{2}} \leq\left\|(-\triangle)^{(2-s) / 2}(-\triangle+V)^{s-1} f\right\|_{L^{2}}
$$

and $\left\||V|^{s / 2} f\right\|_{L^{2}} \leq C\|f\|_{\dot{H}^{s}}$. We estimate the right-hand side of (5.7) using (5.4) and find

$$
\left\||V|^{(2-s) / 2}(-\triangle+V)^{s-1} f\right\|_{L^{2}} \leq C\left\|(-\triangle+V)^{s / 2} f\right\|_{L^{2}} .
$$

From (5.4), (5.7) and (5.8) we obtain

$$
\left|I_{2}\right| \leq C\|f\|_{\dot{H}_{V}^{s}}\|f\|_{\dot{H}^{s}} .
$$

This estimate, (5.4) and (5.3) lead to

$$
\|f\|_{\dot{H}_{V}^{s}}^{2} \leq C\|f\|_{\dot{H}_{V}^{s}}\|f\|_{\dot{H}^{s}} .
$$


Hence

$$
\|f\|_{\dot{H}_{V}^{s}} \leq C\|f\|_{\dot{H}^{s}}
$$

for $0 \leq s<\frac{3}{2}$. To show the opposite inequality, we modify (5.3) as follows:

$$
\begin{gathered}
\left\|(-\triangle)^{s / 2} f\right\|_{L^{2}}^{2}=\left((-\triangle)^{s-1} f,(-\triangle) f\right)_{L^{2}} \\
=\left((-\triangle)^{s-1} f,(-\triangle+V) f\right)_{L^{2}}-\left((-\triangle)^{s-1} f, V f\right)_{L^{2}} \\
=\left((-\triangle+V)^{1-s / 2}(-\triangle)^{s-1} f,(-\triangle+V)^{s / 2} f\right)_{L^{2}} \\
-\left(|V|^{1-s / 2}(-\triangle)^{s-1} f, \operatorname{sgn} V|V|^{s / 2} f\right)_{L^{2}} .
\end{gathered}
$$

Let we set

$$
\begin{gathered}
I_{3}=\left((-\triangle+V)^{1-s / 2}(-\triangle)^{s-1} f,(-\triangle+V)^{s / 2} f\right)_{L^{2}}, \\
I_{4}=\left(|V|^{1-s / 2}(-\triangle)^{s-1} f, \operatorname{sgn} V|V|^{s / 2} f\right)_{L^{2}} .
\end{gathered}
$$

Since $2-s \in(0,1)$ we can use Lemma 5.1 and find $\left\|(-\triangle+V)^{(2-s) / 2}(-\triangle)^{s-1} f\right\|_{L^{2}}$ $\leq C\left\|(-\triangle)^{s / 2} f\right\|_{L^{2}}$, so $I_{3} \leq C\|f\|_{\dot{H}_{V}^{s}}\|f\|_{\dot{H}^{s}}$. For the term $I_{4}$ we apply Lemma 5.2 and find $\left|I_{4}\right| \leq C \delta_{0}^{s / 2}\|f\|_{\dot{H}^{s}}^{2}$. So for $\delta_{0}>0$ small enough we get $\|f\|_{\dot{H}^{s}}^{2} \leq$ $C\|f\|_{\dot{H}^{s}}\|f\|_{\dot{H}_{V}^{s}}+C \delta_{0}\|f\|_{\dot{H}^{s}}^{2}$ and $\|f\|_{\dot{H}^{s}} \leq C\|f\|_{\dot{H}_{V}^{s}}$. Thus we complete the proof of Theorem 1.4 .

\section{REFERENCES}

1. Bergh, J. , Löfström, J., Interpolation spaces, Springer-Verlag, 1976. MR0482275 (58:2349)

2. Burq, N., Planchon, F., Stalker, J. and Shadi Tahvildar-Zadeh, Strichart estimates for the Wave and Schrödinger Equations with the Inverse-Square Potential , Preprint, 2002.

3. Engel, K.-J., Nagel, R. One-parameter semigroups for linear evolution equations, Graduate Texts in Mathematics, 194. Springer-Verlag, New York, 2000. MR1721989 (2000i:47075)

4. Ginibre, J., Velo, G. Generalized Strichartz inequalities for the wave equation, J. Funct. Anal. 133 (1995), 50-68. MR.1351643 (97a:46047)

5. Jensen, A., Kato, T. Spectral properties of Schrödinger operators and time-decay of the wave functions. Duke Math. J. 46 (1979), no. 3, 583-611. MR0544248 (81b:35079)

6. Journé, J.-L., Soffer, A., Sogge, C. D. Decay estimates for Schrödinger operators. Comm. Pure Appl. Math. 44 (1991), no. 5, 573-604. MR 1105875|(93d:35034)

7. Jensen, A., Nakamura, S. Mapping properties of functions of Schrödinger operators between $L^{p}$-spaces and Besov spaces. Spectral and scattering theory and applications, 187-209, Adv. Stud. Pure Math., 23, Math. Soc. Japan, Tokyo, 1994. MR1275402 (96a:47084)

8. Jensen, A., Nakamura, S. $L^{p}$-mapping properties of functions of Schrödinger operators and their applications to scattering theory. J. Math. Soc. Japan 47 (1995), no. 2, 253-273. MR $1317282(95 \mathrm{~m}: 47087)$

9. Keel, M., Tao, T. Endpoint Strichartz estimates. Amer. J. Math. 120 (1998), no. 5, 955-980. MR1646048 (2000d:35018)

10. O'Neil, R. Convolution operator and $L^{p, q}$ - spaces. Duke Math.J. 30, 1963, 129 - 142. MR0146673 (26:4193)

11. Reed, M., Simon, B. Methods of Modern Mathematical Physics, vol.2, Academic Press, 1975. MR 0493420 (58:12429b)

12. Rodnianski, I., Schlag, W.Time decay for solutions of Schrödinger equations with rough and time-dependent potentials, preprint 2002.

13. Shibata, Y., Tanaka, K.On the steady flow on compressible viscous fluid and its stability with respect to initial disturbance. J.Math.Soc.Japan 55 (2003), No.3, 797 - 826. MR 1978223 (2004b:76127) 
14. Simon, B. Schrödinger semigroups. Bull. Amer. Math. Soc. (N.S.) 7 (1982), no. 3, 447-526. MR0670130 (86b:81001a)

15. Zeidler, E. Nonlinear functional analysis and its applications. I. Fixed-point theorems. Springer-Verlag, New York, 1986. MF 0816732 (87f:47083)

Dipartimento di Matematica, Università di Pisa, Via Buonarroti No.2, 56127 - Pisa, ITALY

E-mail address: georgiev@dm.unipi.it

Dipartimento di Matematica, Università di Pisa, Via Buonarroti No.2, 56127 - Pisa, ITALY

E-mail address: ivanov@mail.dm.unipi.it 